\title{
A virtual, three-dimensional temporal bone model and its educational value for neurosurgical trainees
}

Peter J. Morone, MD, MSCI, ${ }^{1}$ Kushal J. Shah, MD, ${ }^{2}$ Benjamin K. Hendricks, MD, ${ }^{3}$ Aaron A. Cohen-Gadol, MD, MSc, MBA ${ }^{4}$

${ }^{1}$ Vanderbilt University Medical Center, Department of Neurological Surgery, $116121^{\text {st }}$ Avenue South, Suite T-4224 MCN, Nashville, TN 37232-2380, USA

2University of Kansas Medical Center, Department of Neurosurgery, Kansas City, KS 66160, USA

${ }^{3}$ Barrow Neurological Institute, Department of Neurological Surgery, 350 West Thomas Rd, Phoenix, AZ 85013 USA

${ }^{4}$ Indiana University School of Medicine, Department of Neurological Surgery, 355 West $16^{\text {th }}$

Street, Suite 5100, Indianapolis, IN 46202, USA

Email addresses:

peter.morone@vanderbilt.edu

kushalshah@mail.umkc.edu

ben.k.hendricks@me.com

acohenmd@gmail.com

\section{Corresponding Author}

Aaron Cohen-Gadol, MD, MSc, MBA

Goodman Campbell Brain and Spine

Indiana University Department of Neurosurgery

$355 \mathrm{~W} \mathrm{16}{ }^{\text {th }}$ Street

Suite 5100

Indianapolis, IN 46202

Phone: 317-362-8760

Fax: 317-924-8472

E-mail: acohenmd@gmail.com

Key Words: anatomy; neurosurgery; three-dimensional; temporal bone; virtual reality

Running Title: A virtual temporal bone model

Financial Support: the authors received no financial support for this research and have no conflicts of interest related to this study.

Abstract word count: 213

Text word count: 1555

Number of references: 24

Number of tables and/or figures (total); 6 (3 tables and 3 figures)

Number of videos: 0 
Morone et al. $\quad 2$

\section{Abstract}

1 Objective: Learning complex neuroanatomy is an arduous yet important task for every neurosurgical trainee. As technology has advanced, various modalities have been created to aid

3 our understanding of anatomy. This study sought to assess the educational value of a virtual,

4 three-dimensional (3D) temporal bone model.

5 Methods: The 3D temporal bone model was created with assistance of computer graphic

6 designers and published online. Its educational value as a teaching was tool was assessed by

7 querying 73 neurosurgery residents at four institutions and was compared to that of a standard,

8 two-dimensional (2D) temporal bone resource. Data was collected via a survey and significance

9 amongst responses was analyzed via a univariate chi-square test.

10 Results: The survey response rate was $37 \%$. Greater than $90 \%$ of residents preferred to study

11 with the 3D model compared with the 2D resource and felt that the 3D model allowed them 12 understand the anatomy more realistically $(\mathrm{p}=.001)$. Moreover, greater than $90 \%$ of residents 13 believed that reviewing the 3D model prior to an actual surgery could lead to improved operative 14 efficiency and safety $(\mathrm{p}=.001)$.

15 Conclusions: This study demonstrates the utility of a novel, 3D temporal bone model as a 16 teaching tool for neurosurgery residents. The model contains accurate anatomic structures and 17 allows user interaction via a virtual, immersive environment. 


\section{Introduction}

Understanding complex neurosurgical anatomy is an important task that takes years to master. Throughout history, neurosurgeons have built a library of resources allowing neurosurgical trainees to familiarize themselves with anatomy prior to entering the operating room. These resources improve our understanding of operative space and allow us to move confidently through an operation.

From Cushing to Rhoton, visualization of anatomy has advanced from simple sketches to detailed, masterfully prepared cadaveric specimens.(1-5) Today, as technology continues to advance, a host of virtual reality models have been designed to help trainees understand operative anatomy,(6-8) yet this technology is young and has the potential to be continually improved.

In this study, our goal was to create a novel, three-dimensional (3D) temporal bone model that users could examine and interact through a virtual reality space. Additionally, we sought to evaluate the models' effectiveness as a teaching tool by comparing it with a two-dimensional (2D) temporal bone resource.

\section{Methods}

3D Model \& 2D Resource Development

The 3D temporal bone model was built by collecting imaging data and working in conjunction with computer graphic designers. First, a 3D MRI (slice thickness $1.10 \mathrm{~mm}, 1.5$ Tesla scanner) and CT scan (slice thickness, 0.625, 64 slice scanner, General Electric $®$ ) of a Caucasian male was obtained. Second, using Amira ${ }^{\circledR}$ (Thermo Fisher Scientific, Waltham, Massachusetts), MRI data was converted into a polygonal mesh model, which was exported into Maya ${ }^{\circledR}$ (Autodesk, 
San Rafael, California), a 3D computer graphics modeling software. Third, the model underwent rendering with Zbrush ${ }^{\circledR}$ (Pixologic, Los Angeles, California), allowing designers to subject the model to high-resolution, digital sculpting. Fourth, for a more realistic appearance, the model was painted and textures were added. Finally, once the desired appearance was achieved, the finalized 3D model was uploaded to Sketchfab® (Sketchfab, New York, New York), an online 3D content publishing platform.

The model, which can be accessed for free online,(9) contains 48 anatomical structures that can be explored by the user (Fig. 1). The model can be rotated and viewed in multiple dimensions, and users have the capability to zoom in and out to examine various structures and anatomical relationships. Currently, the model can be visualized on a mobile phone, personal computer, or a virtual reality headset that allows an immersive experience.

The reference included to represent 2D models was created by combining resources commonly utilized by neurosurgery residents, including select illustrations from Atlas of Human Anatomy,(10) Thieme Atlas of Anatomy - Head, Neck, and Neuroanatomy(11) and Atlas of the Human Skull.(12) There were also links provided to 13 relevant illustrations available through the online Albert L. Rhoton image database.(10) These resources were provided in a quick reference view to minimize the distraction of irrelevant content during the evaluation process. Study Design

To compare the 3D and 2D resources, a 9-question survey (see Supplemental Materials) was created using RedCap ${ }^{\circledR}$ software.(13) On January 11, 2018, the survey was sent by the authors to all neurosurgery residents at the following institutions: Indiana University School of Medicine Department of Neurosurgery, Vanderbilt University Medical Center, Kansas University Medical Center, and the Barrow Neurological Institute. A total of 73 residents met eligibility criteria and 
were e-mailed the survey along with links to the 3D and 2D resources. Residents were then asked to review both resources and complete the survey. The survey was closed on February 8 , 2018.

\section{Statistical Analysis}

Survey questions were analyzed using descriptive statistics. Statistical significance was assessed via a univariate chi-square test, and a two-sided $\mathrm{P}$ value of less than .05 was considered to indicate significance. Data analysis was completed using Stata (version 15.1).

\section{Results}

The survey response rate was $37 \%$ ( 27 of 73 participants), and the year of residency for each participant can be seen in Table 1 .

The majority of participants $(96 \%, \mathrm{p}=.001)$ preferred studying with the $3 \mathrm{D}$ model versus the 2D resource. Additionally, they felt that it was easier to learn the complex temporal bone anatomy when reviewing the 3D model $(93 \%, \mathrm{p}=.001)$ (Table 2).

Moreover, 23 participants "strongly agreed" or "agreed" that the 3D model demonstrated more realistic anatomical structures (Fig. 2), and 24 participants "strongly agreed" or "agreed" that the 3D model depicted more accurate anatomical relationships compared with the 2D resource (Fig. 3).

A significant number of participants felt that reviewing the 3D model prior to an operative case would improve both operative efficiency $(96 \%, \mathrm{p}=.001)$ and operative safety $(93 \%, \mathrm{p}=.001)$ (Table 3). Finally, 96\% of participants would like future access to 3D models for other neuroanatomical structures (Table 3). 


\section{Discussion}

In this study, we created a novel, 3D resource of the temporal bone. We then asked neurosurgery residents at four institutions to compare this 3D model with a 2D resource and complete a 9question online survey pertaining to both resources' educational value. Overall, as expected, we found that residents preferred the $3 \mathrm{D}$ resource compared with the $2 \mathrm{D}$ resource. The participants found the $3 \mathrm{D}$ resource a more instructive representation of the temporal bone anatomy and thought that it would improve both operative efficiency and safety when used to prepare for a neurosurgical case. Finally, most residents agreed that the 3D resource depicted more accurate anatomical structures and relationships compared with the 2D resource (Figs 2, 3).

As technology advances, we should use it to our advantage to create educational tools that can be used to improve surgical training and immersive imagination. Certainly, technology from hand-drawn sketches to cadaveric dissections to advanced virtual reality 3D models - has facilitated the evolution of anatomical learning. Around $300 \mathrm{BC}$, Euclid was one of the first to describe binocular vision through his theories of geometry and optics.(14) It was not until the Renaissance that Brunelleschi uncovered a system of linear perspective that allowed artists to depict 3D scenes, which Leonardo Da Vinci and Vesalius further advanced.(15) One of the first actual 3D images was created in the 1830s, when Charles Wheatstone invented the stereoscope, which depicted separate images for the left and right eye of the same scene.(15) This eventually led to the first collection of widely available stereoscopic anatomy plates in the Edinburgh Stereoscopic Atlas of Anatomy.(15) Additionally, in 1894, Edward Flatau, a Polish neurologist created a detailed photographic atlas of the unfixed human brain. (16) In the early 1900s, Max Brödel, a German artist, assisted Harvey Cushing in illustrating the transsphenoidal approach, (3) and in 1911, he founded the Department of Art as Applied to medicine at Johns Hopkins 
111 University $(17,18)$ which has trained more than 200 medical illustrators,(3) including Dorcas

112 Hager Padget who worked closely with Walter Dandy.(19) In 1993, Dr. Albert Rhoton translated

113 2D microanatomical dissections into masterpieces of 3D images by using stereophotography and

114 polarized glasses.(20) The AANS has made Dr. Rhoton's lectures available in 3D formats to

115 neurosurgeons worldwide and this technology continues to improve.(15)

116 In 1999, D’Urso et al(21) created intracranial vascular biomodels using

117 stereolithography, a method that has currently been advanced to print 3D models, including a

118 temporal bone model that can be drilled.(22) Other 3D models that have been created include a

119 transsphenoidal model for resecting pituitary lesions, a ventricular model for placement of an

120 external ventricular drain, and multiple spine models used for placement of pedicle screws.

121 (23)'(24) Importantly, 3D modeling has been tested and shown to improve resident

122

123

124

125

126

127 understanding of various anatomical landmarks. (23)

We built upon the current 3D technology by creating a high-fidelity 3D model that users can manipulate and interact with in a virtual environment. Our temporal bone model allows users to examine it from any viewpoint in space. Additionally, users have the ability to zoom in and out, allowing them to survey anatomy at different magnifications. This feature yields views that are similar to the operative environment while physicians perform surgery under the microscope. Furthermore, our model is color-coated and labeled with 48 different anatomic landmarks that users can click on and visualize at different angles. Overall, this model provides an accurate and immersive experience for the user, allowing them to actively engage in the temporal bone space. We believe this type of learning format is the next generation of anatomical visualization and may improve knowledge retention, which could ultimately translate to improved surgical performance. 
Our study does have several limitations and could be improved. First, a sample size of more than 27 residents would improve the strength of our data. Second, our survey was targeted to obtain subjective data about the educational value of the 3D and 2D resources. Since our goal was to obtain participants' opinions about the resources, we decided this type of survey design was ideal for our pilot study. However, a more objective educational value of the 3D resource compared with the $2 \mathrm{D}$ resource could be better elucidated by obtaining quantitative data. For example, a stronger study would ask a random group of users to review either the 3D or 2D resource and then have them take an exam to assess their knowledge of temporal bone anatomy.

The results of the exam could be used to calculate a quantifiable difference between both resources. In the future, we hope to complete larger studies with stronger, measurable results.

\section{Conclusions}

We constructed a novel, interactive 3D temporal bone model and queried neurosurgery residents to evaluate its educational value via a survey. We discovered that nearly every survey participant preferred the 3D model compared with the 2D resource. Although future, larger studies will help determine the 3D model's full education value, this study demonstrates its utility as a learning tool amongst neurosurgical trainees.

\section{References}

1. $\quad$ Ellis H. Harvey Williams Cushing (1869-1939). J Med Biogr. 1994;2(2):71-7.

2. Scholz M, Parvin R, Thissen J, Lohnert C, Harders A, Blaeser K. Skull base approaches in neurosurgery. Head Neck Oncol. 2010;2:16.

3. Patel SK, Couldwell WT, Liu JK. Max Brodel: his art, legacy, and contributions to neurosurgery through medical illustration. J Neurosurg. 2011;115(1):182-90.

4. Matsushima T, Richard Lister J, Matsushima K, de Oliveira E, Timurkaynak E, Peace DA, et al. The history of Rhoton's Lab. Neurosurg Rev. 2017. 
5. Matsushima T, Matsushima K, Kobayashi S, Lister JR, Morcos JJ. The microneurosurgical anatomy legacy of Albert L. Rhoton Jr., MD: an analysis of transition and evolution over 50 years. J Neurosurg. 2018:1-11.

6. Morone PJ, Bekelis K, Root BK, Singer RJ. Development and Validation of a Mobile Device-based External Ventricular Drain Simulator. Oper Neurosurg (Hagerstown). 2017;13(5):603-8.

7. Tucker AM, Beckett JS, Martin NA. Next Generation Case Report: Supraorbital Craniotomy for Anterior Communicating Artery Aneurysm Clipping in Annotated Virtual Reality Environment. Oper Neurosurg (Hagerstown). 2017.

8. Gmeiner M, Dirnberger J, Fenz W, Gollwitzer M, Wurm G, Trenkler J, et al. Virtual Cerebral Aneurysm Clipping with Real-Time Haptic Force Feedback in Neurosurgical Education. World Neurosurg. 2018;112:e313-e23.

9. Temporal Bone and Deep Structures Sketchfab: https://sketchfab.com/models/8958d2d938734a6db18c24652561b0fe.; [Available from: https://sketchfab.com/models/8958d2d938734a6db18c24652561b0fe.

10. $\quad$ FH N. Atlas of Human Anatomy: Elsevier - Health Sciences Division; 2014. 11. Schuenke M SE, Schumacher U. Head, Neck, and Neuroanatomy (THIEME Atlas of Anatomy): Thieme; 2016.

12. Sampson HW MJ, Henryson GL. Atlas of the Human Skull: Texas A\&M University Press; 1991.

13. Harris PA, Taylor R, Thielke R, Payne J, Gonzalez N, Conde JG. Research electronic data capture (REDCap)--a metadata-driven methodology and workflow process for providing translational research informatics support. J Biomed Inform. 2009;42(2):377-81.

14. Burton H. The optics of Euclid. J Optical Soc Am. 1945;35:357-72.

15. Robertson JH, Sorenson JM. The visual dimensions of future neurosurgical education. World neurosurgery. 2011;76(6):492-6.

16. Triarhou LC. A review of Edward Flatau's 1894 Atlas of the Human Brain by the neurologist Sigmund Freud. Eur Neurol. 2011;65(1):10-5.

17. Brodel M. Medical Illustration. JAMA : the journal of the American Medical Association. 1941;117:668-72.

18. Cullen TS. Max Brödel, 1870-1941 Director of the First Department of Art as Applied to Medicine in the World. Bulletin of the Medical Library Association. 1945;33(1):4.1-29.

19. Sugar O. Dorcas Hager Padget: artist and embryologist. Surg Neurol. 1992;38(6):464-8. 20. Martins C, Ribas EC, Rhoton AL, Jr., Ribas GC. Three-dimensional digital projection in neurosurgical education: technical note. J Neurosurg. 2015;123(4):1077-80.

21. D'Urso PS, Thompson RG, Atkinson RL, Weidmann MJ, Redmond MJ, Hall BI, et al. Cerebrovascular biomodelling: a technical note. Surgical neurology. 1999;52(5):490-500.

22. Wanibuchi M, Noshiro S, Sugino T, Akiyama Y, Mikami T, Iihoshi S, et al. Training for Skull Base Surgery with a Colored Temporal Bone Model Created by Three-Dimensional Printing Technology. World neurosurgery. 2016;91:66-72.

23. Shah KJ, Peterson JC, Beahm DD, Camarata PJ, Chamoun RB. Three-Dimensional Printed Model Used to Teach Skull Base Anatomy Through a Transsphenoidal Approach for Neurosurgery Residents. Operative Neurosurgery. 2016;12(4):326-9.

24. Chan S, Conti F, Salisbury K, Blevins NH. Virtual reality simulation in neurosurgery: technologies and evolution. Neurosurgery. 2013;72 Suppl 1:154-64. 
Morone et al. 10

\section{Figure Legends}

207 Fig. 1. 3D Model of the temporal bone viewed lateral (left) and medial (right) surfaces. The 208 model can be freely rotated and viewed in multi-dimensions online

209 (https://www.neurosurgicalatlas.com/volumes/cranial-base-surgery/skull-base-

210 exposures/anterior-petrosectomy). With Permission from The Neurosurgical Atlas by Aaron 211 Cohen-Gadol, MD.

213 Fig. 2. Bar graph showing participants' responses. The x-axis represents the type of response on 214 a Likert-type scale and the y-axis represents the number of responses.

216 Fig. 3. Bar graph showing participants' responses. The x-axis represents the type of response on 217 a Likert-type scale and the y-axis represents the number of responses. 
Table 1: Participants' Year in Residency

\begin{tabular}{lc}
\hline Postgraduate Year & Number $(\%)$ of Residents \\
\hline 1 & $5(18)$ \\
2 & $4(15)$ \\
3 & $4(15)$ \\
4 & $2(7)$ \\
5 & $8(30)$ \\
6 & $4(15)$ \\
\hline
\end{tabular}




\begin{tabular}{lccc}
\hline & $3 \mathrm{D}(\%)$ & $2 \mathrm{D}(\%)$ & $\mathrm{p}$ Value \\
\hline $\begin{array}{l}\text { If given a choice, would you choose to study } \\
\text { anatomy using the 2D or 3D resource? }\end{array}$ & $26(96)$ & $1(4)$ & .001 \\
$\begin{array}{l}\text { Do you think it is easier to learn anatomy using the } \\
\text { 2D or 3D resource? }\end{array}$ & $25(93)$ & $2(7)$ & .001 \\
\hline
\end{tabular}


Table 3: Operative Applicability

\begin{tabular}{lccc}
\hline & No. (\%) Yes & No. (\%) No & p Value \\
\hline $\begin{array}{l}\text { Do you feel that reviewing a 3D resource prior to } \\
\text { an OR case would improve your operative }\end{array}$ & $26(96)$ & $1(4)$ & .001 \\
$\begin{array}{l}\text { efficiency? } \\
\begin{array}{l}\text { Do you feel that reviewing a 3D resource prior to } \\
\text { an OR case would improve your operative safety? }\end{array}\end{array} 25(93)$ & $2(7)$ & .001 \\
$\begin{array}{l}\text { Would you like to have access to a 3D resource for } \\
\text { all neuroanatomical structures? }\end{array}$ & $26(96)$ & $1(4)$ & .001 \\
\hline
\end{tabular}




\section{ACCEPTED MANUSCRIPT}
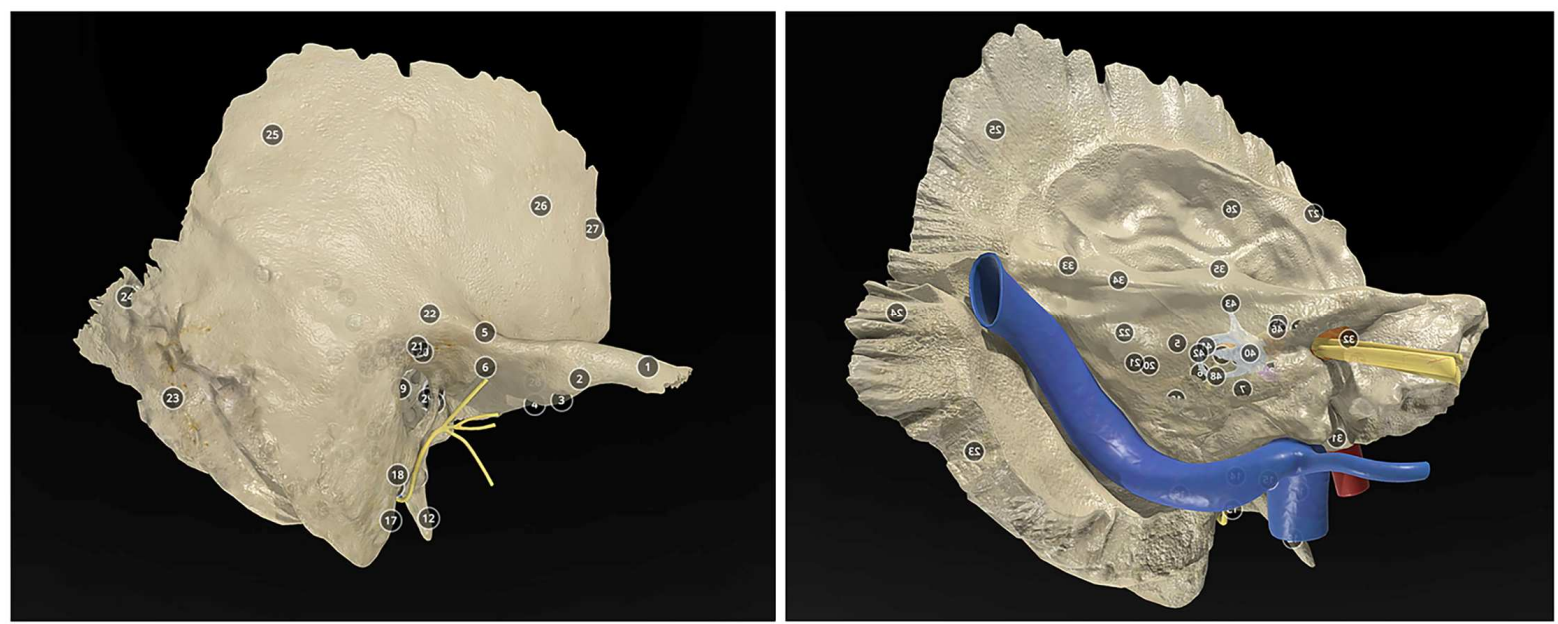
Compared to the 2D resource, do you feel that the 3D resource depicts more realistic anatomical structures?

14

12

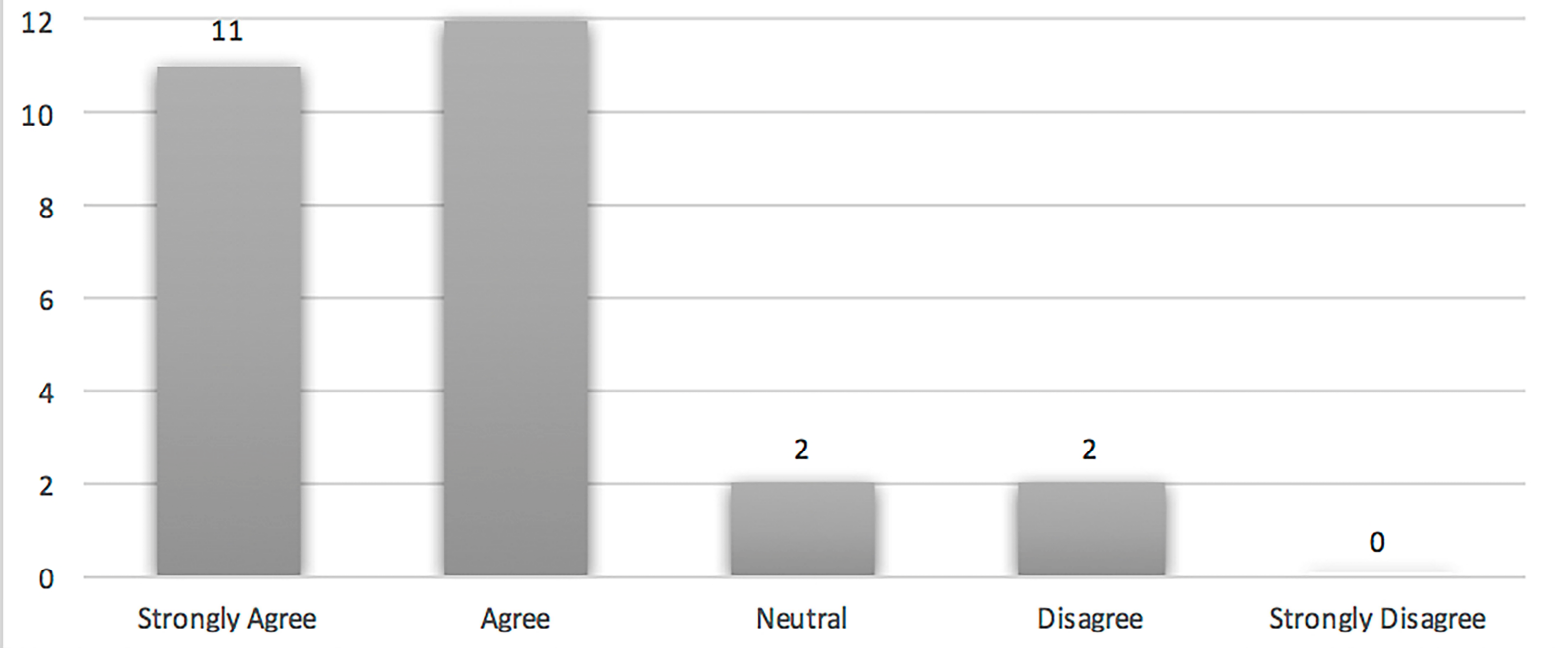




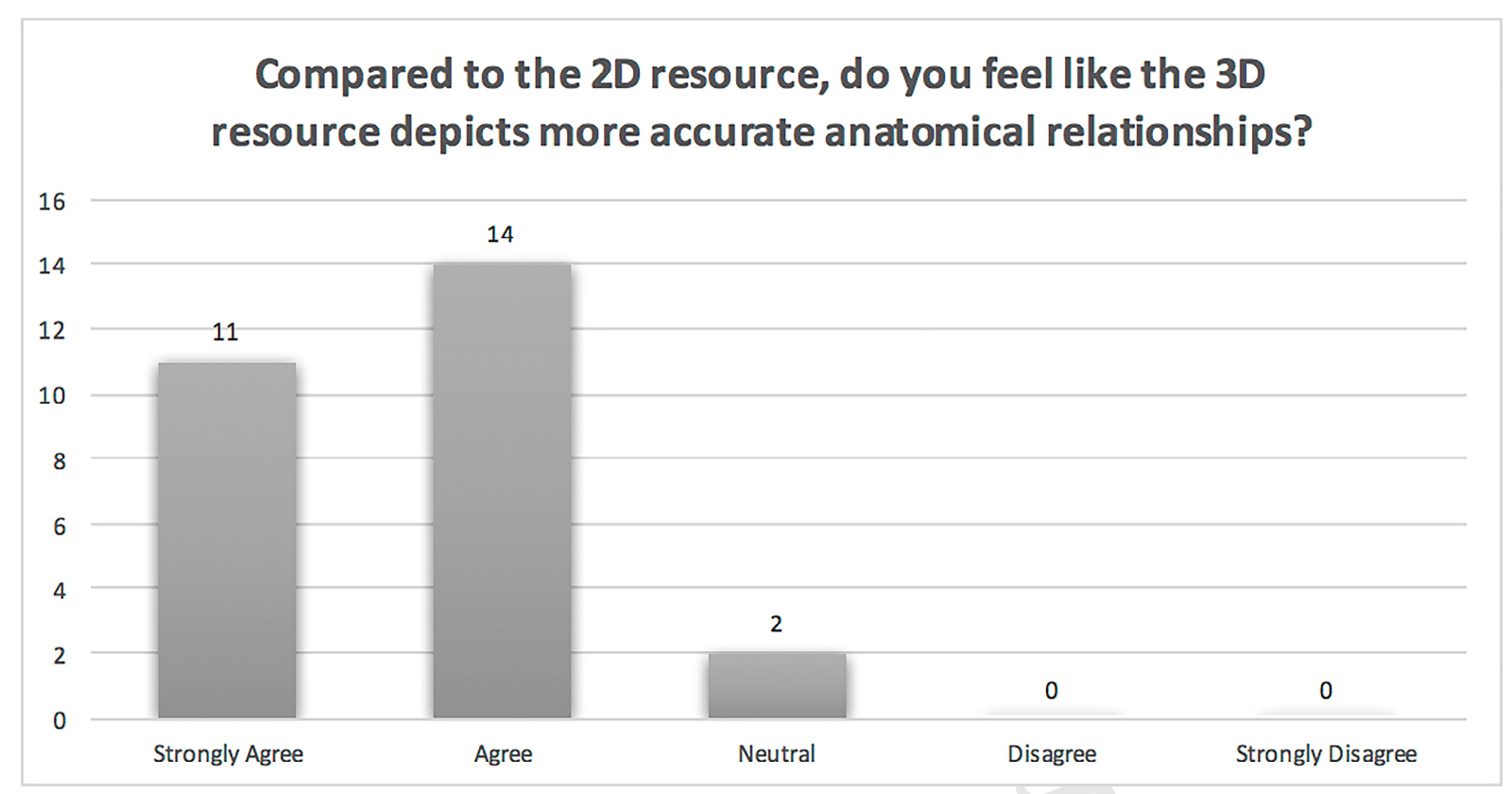




\section{Abbreviations}

2D, two-dimensional

3D, three-dimensional 